\title{
Electrospun Pitch/Polyacrylonitrile composite carbon nanofibers as high performance anodes
}

\section{for lithium-ion batteries}

\author{
Zhiqiang Shi ${ }^{\mathrm{a}, *}$, Chuanbin Chong ${ }^{\mathrm{a}}$, Jing Wang ${ }^{\mathrm{a}}$, Chengyang Wang ${ }^{\mathrm{b}}$, Xuewen $\mathrm{Yu}^{\mathrm{a}}$ \\ ${ }^{a}$ Laboratory of Fiber modification and Functional Fiber, College of Materials Science and Engineering, \\ Tianjin Polytechnic University, Tianjin 300387, P. R. China \\ ${ }^{\mathrm{b}}$ Key Laboratory for Green Chemical Technology of Ministry of Education, School of Chemical \\ Engineering and Technology, Tianjin University, Tianjin 300072, P. R. China
}

\begin{abstract}
A new kind of soft carbon and hard carbon composite nanofibers were fabricated from isotropic pitch and polyacrylonitrile via simple electrospinning followed by stabilization and carbonization. The obtained fibrous mat was directly used as anodes of lithium-ion batteries without binder added and current collector. The composite nanofibers electrodes display a large reversible capacity of $452 \mathrm{mAh} \mathrm{g}^{-1}$ at a current density of $20 \mathrm{~mA} \mathrm{~g}^{-1}$ and a capacity of $255 \mathrm{mAh} \mathrm{g}^{-1}$ at $200 \mathrm{~mA} \mathrm{~g}^{-1}$ after 200 cycles. The improved electrochemical performance can be attributed to the unique fibrous structure which will facilitate electrons and ions transfer and the porous structure which will accommodate quantities of lithium ions.
\end{abstract}

Keywords: Electrospinning; Carbon materials; Composite nanofibers; Energy storage and conversion

\section{Introduction}

Rechargeable Lithium-ion batteries (LIBs) have attracted extensive attention due to their wide applications in small-sized portable electronics and potential application in electric/hybrid vehicles [1,2].

"Corresponding author. Tel.: +86 22 83955816;

E-mail address: shizhiqiang@ @ tjpu.edu.cn (Z.Q. Shi) 
Currently, most of the commercial LIBs use graphite as the anode material. Graphite suffers from the inherently low theoretical capacity $\left(372 \mathrm{mAh} \mathrm{g}^{-1}\right)$, poor cycle performance and rate capability. All the drawbacks above restrict its application in electric/hybrid vehicles. So it is crucial to develop electrode material with both higher energy and power density.

Hard carbon materials contain a large amount of nano-sized pores, which consisted of small single graphene sheets arranged like a house of cards [3]. The theoretical capacity of hard carbon material reaching $740 \mathrm{mAh} \mathrm{g}^{-1}$, which is approximately twice of graphite material, for the reason that the lithium ions could adsorb on both sides of these single layers and embed in nanopores [4]. However, hard carbon shows poor rate performance and cycle stability resulting from their relatively low conductivity for the highly disordered structure. As another carbonaceous material, soft carbon contains small crystallite domains where a few graphene sheets are stacked approximately parallel to each other. Attributed to this distinctive microstructure, soft carbon shows excellent cycle performance and good rate capability [3, 5].

Herein, we choose isotropic pitch as precursor of soft carbon and polyacrylonitrile (PAN) as precursor of hard carbon and prepared a soft carbon/hard carbon composite nanofibers through a simple electrospinning method. After stabilization and carbonization, carbonized nanofibers (CNFs) web were flexible and were directly used as anode material for lithium-ion batteries. The ground carbonized nanofibers (G-CNFs) were presented as a comparison. The CNFs possess a synthesis electrochemical characteristics of hard carbon and soft carbon materials, display a large reversible capacity, excellent cycle performance and good rate capability. All these superior properties are attributed to the unique structure and the 3-dimentional connected web, which enhanced ion diffusion and electron transfer. 


\section{Experimental}

Material preparation: Pitch was dissolved in a binary solvent (THF:DMF=2:1) to form a $40 \mathrm{wt} \%$ solution by vigorous stirring at $60^{\circ} \mathrm{C}$ for $12 \mathrm{~h}$. Then a previously prepared solution of PAN (molecular weight=160000) in DMF was added to pitch solution to achieve a Pitch/PAN weight ratio of 5/5 wt\%. This mixture was continuously stirred until a homogeneous solution was obtained. Electrospinning was carried out to produce pitch/PAN composite nanofibers with a $1.5 \mathrm{mlh}^{-1}$ flow rate, $15 \mathrm{~cm}$ needle to collector distance and $20 \mathrm{kV}$ voltages between needle and collector. The as-collected fibers were first stabilized in an air atmosphere at $280^{\circ} \mathrm{C}$ for $1 \mathrm{~h}$ and then carbonized at $900^{\circ} \mathrm{C}$ for $2 \mathrm{~h}$ in nitrogen.

Material characterizations: Field-emission scanning electron microscopy (FESEM, Hitachi S-4800) was used to observe the morphology of the fibers. Transmission electron microscopy (TEM) and high-resolution transmission electron microscopy (HR-TEM) images were recorded using a Tecnai G2 F20 TEM. X-ray diffraction was carried out on a Rigaku D/Max2500 X-ray diffractometer using Cu K $\alpha$ radiation and Raman spectrum on a Renishaw in Via reflex Raman microscope with 523 nm line of Ar-ion laser.

Electrochemical characterizations: Electrochemical performance evaluation was performed using lithium-ion half cells. Two methods were used to prepare the working electrodes. One is that the free-standing nanofibers mat was cut into disks to be directly used as working electrodes and denoted as CNFs. In another method, the nanofibers mat was ground into powder and mixed with acetylene black (10\% weight) and polyvinylidene fluoride (10\% weight), and then coated onto a copper foil. Electrodes made in this method were denoted as G-CNFs. $\operatorname{LiPF}_{6}(1 \mathrm{M})$ in ethylene carbonate/dimethyl carbonate (EC/DMC, 1:1 by volume) was employed as the electrolyte. Galvanostatic charge-discharge experiments 
were conducted using a multichannel Land Battery Test System over the range of 0.005-2.5 V. Electrochemical impedance spectroscopy (EIS) measurement was performed on a CHI604d electrochemical workstation.

\section{Results and discussion}

Fig. 1a and $\mathrm{b}$ show the FESEM images of as-spun Pitch/PAN composite nanofibers web and carbonized nanofibers web, respectively. As we can see, the as-spun fibers are long, continuous and randomly oriented, building a 3-dimentional interconnected network with a uniform diameter ranging from 300 to $400 \mathrm{~nm}$. After heat treatment in nitrogen, the carbonized nanofibers (CNFs) maintain the fibrous morphology and the interconnected network were kept perfectly (Fig. 1b), which will be beneficial for both the electron and ion transfers. The average diameter of CNFs decreases slightly due to removal of hydrogen, oxygen, and nitrogen elements during the carbonization process [6]. The carbonized fiber films were flexible and can be bended as shown in inset of Fig. 1b. TEM image (Fig. 1c) shows a rough surface for CNFs indicate that there are some structure defects on the fibers surface. These structure defects provided a large interfacial area between the electrolyte and the active material and functioned as active sites for Li ion storage [7]. HR-TEM image (Fig. 1d) shows that CNFs were poorly crystallized and contained many nanocrystalline domains where a few small short graphene sheets stacked in a parallel fashion. There are large quantities of nanopores between these nanocrystalline domains. The nanopores in carbon material act effectively as "reservoir" for Li-ion storage [8, 9]. 

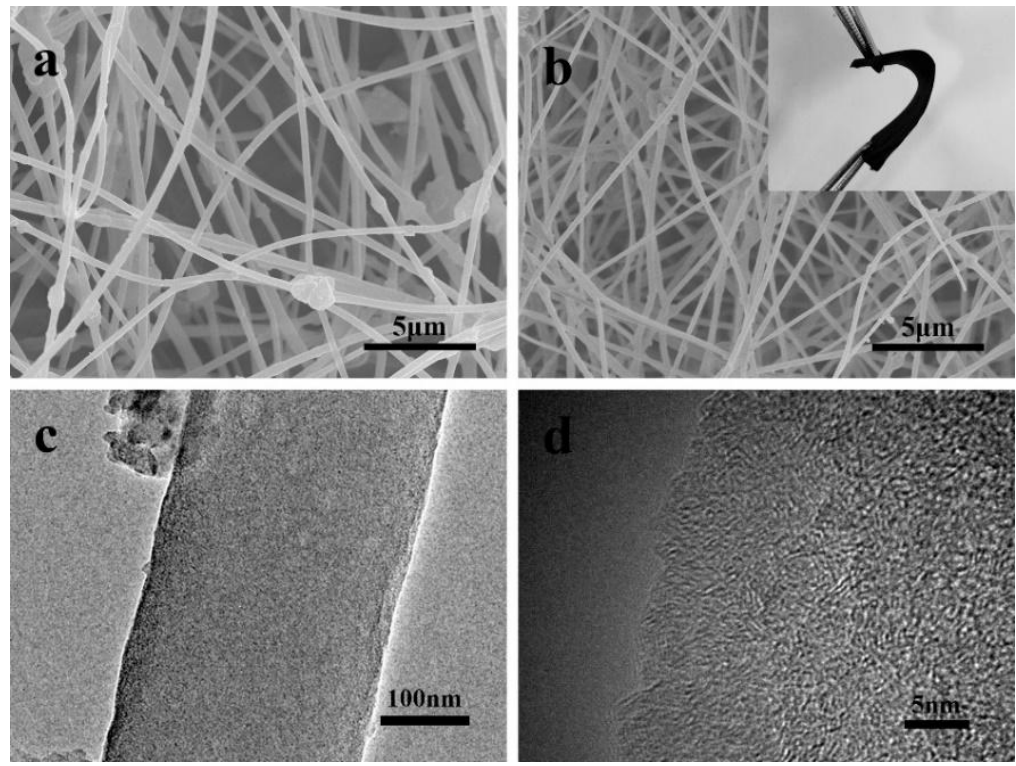

Fig. 1. FESEM images of (a) as-spun fibers and (b) carbonized fibers. The inset of (b) shows the flexible carbonized fiber films. (c) TEM images and (d) HR-TEM images of carbonized fibers.

Raman spectrum and XRD pattern of carbonized fibers are presented in Fig. 2a and b. The Raman spectrum shows two broad bands at around 1349 (D-band) and $1583 \mathrm{~cm}^{-1}$ (G-band), corresponding to a defect-induced mode and the $\mathrm{E}_{2 \mathrm{~g}}$ graphitic mode, which is typical of disordered carbon [10]. XRD pattern consists of two broad peaks at around $24^{\circ}$ and $43^{\circ}$, which are attributed to the (002) and (100) diffraction modes. The patterns are typical of non-graphitic carbon materials with a highly disordered nanocrystalline structure. The calculated interlayer space of $\mathrm{d}_{002}$ is $0.379 \mathrm{~nm}$, significantly larger than the interlayer spacing of Li inserted graphite, which is favorable for insertion and extraction of Li-ion throughout CNFs. The calculated thickness $\left(\mathrm{L}_{\mathrm{c}}\right)$ of the nanocrystalline domains is $1.14 \mathrm{~nm}$, indicating they are composed of 4 stacked graphene layers and is consistent with the HRTEM observation. Furthermore, the peak tail at low Bragg region suggest the existence of nanopores in CNFs [11], as shown in the HRTEM. 

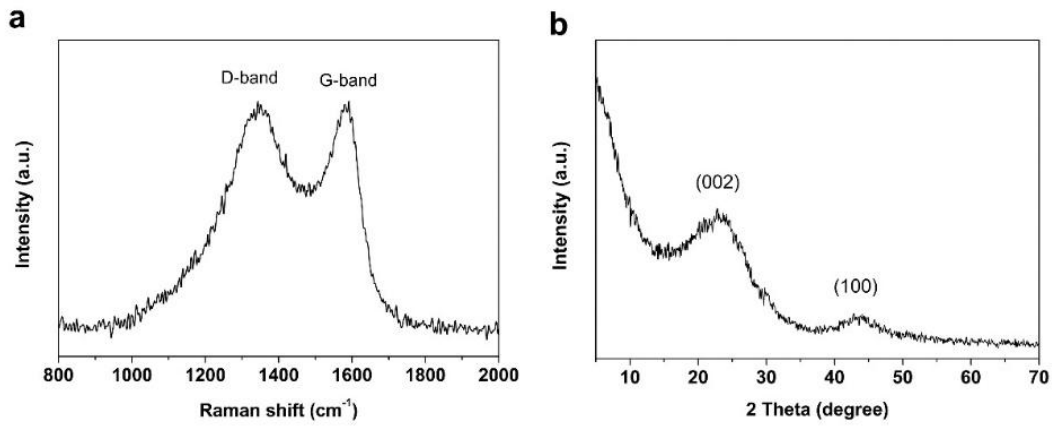

Fig. 2. (a) Raman spectrum and (b) XRD pattern of carbonized fibers.

Fig. 3a shows the initial discharge-charge curves of CNFs and G-CNFs electrodes at a current density of $20 \mathrm{~mA} \mathrm{~g}^{-1}$. Both of the samples show similar discharge-charge curves which consist of a steep slope above $0.2 \mathrm{~V}$ and a plateau at lower potential. The slope region of the profiles corresponds to lithium ions intercalated between turbostratically disordered graphene layers. The capacity of the plateau is attributed to lithium insertion into nanopores among the stacked graphene layers and in the vicinity of residual $\mathrm{H}$ atoms $[3,12]$. The initial discharge capacities of CNFs and G-CNFs are 723 and $505 \mathrm{mAhg}^{-1}$, and charge capacities are 452 and $326 \mathrm{mAh} \mathrm{g}^{-1}$, resulting in an initial Coulombic efficiency of $62.5 \%$ and 64.6\% respectively. The initial irreversible capacity is mainly attributed to the decomposition of electrolytes forming a solid-electrolyte interface (SEI) and lithium irreversible absorbed on the vicinity of residual $\mathrm{H}$ atoms $[13,14]$.

Rate performance and cycle stability of CNFs and G-CNFs are displayed in Fig. 3b and c. The average discharge capacities of CNFs are 423, 349, 285, 234, 210 and $155 \mathrm{mAh} \mathrm{g}^{-1}$ at current densities of $0.02,0.05,0.2,0.4,1$, and $2 \mathrm{~A} \mathrm{~g}^{-1}$ respectively, which are much high than the G-CNFs electrodes. Even at high current density of $4 \mathrm{~A} \mathrm{~g}^{-1}$, CNFs anode can deliver a reversible capacity of $123 \mathrm{mAh} \mathrm{g}^{-1}$, indicating the excellent rate performance. The excellent rate capability is mainly attributed to its unique fibrous structure and interconnected carbonaceous conductive network which will beneficial for both the electron 
and ion transfers. When returned to lower current density of $50 \mathrm{~mA} \mathrm{~g}^{-1}$, the capacity can recover to 340 $\mathrm{mAh} \mathrm{g}^{-1}$, demonstrating good reversibility and structure stability. Fig. $3 \mathrm{c}$ shows the cycle performance of CNFs and G-CNFs tested at $0.2 \mathrm{~A} \mathrm{~g}^{-1}$. After 200 cycles, the CNFs electrode still maintained a reversible capacity of $255 \mathrm{mAh} \mathrm{g}^{-1}$ and correlated capacity retention rate is $91.4 \%$, suggesting excellent cycle stability. G-CNFs also show very stable cycle performance, however, its capacity is much lower than CNFs.

Fig. 3d shows the Nyquist plots of CNFs and G-CNFs electrode before discharge and charge cycles. The typical Nyquist plots consist of a quasi-semicircle in the high-frequency region, which related to the charge transfer processes, and of a quasi-sloping line in the low-frequency region which indicates the impedance caused by the Li diffusion [15]. It can be clearly seen that the charge transfer resistance of CNFs is much smaller than the G-CNFs electrode, indicates a faster kinetic process.

a
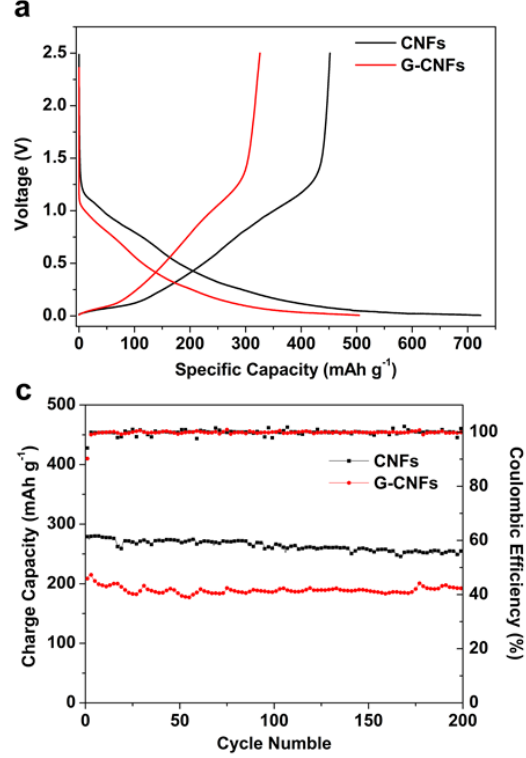
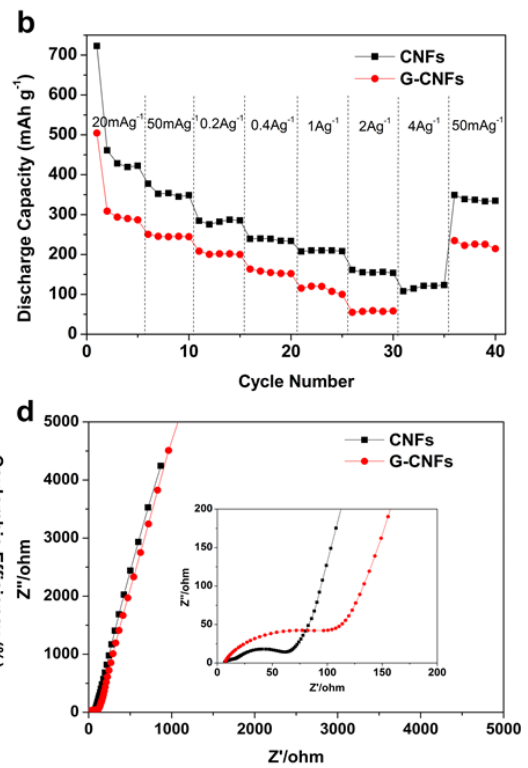

Fig. 3. Electrochemical performance of the CNFs and G-CNFs electrodes. (a) The initial discharge/charge curves at a current density of $20 \mathrm{~mA} \mathrm{~g}^{-1}$. (b) Rate performance. (c) Cycle performance under $0.2 \mathrm{~A} \mathrm{~g}^{-1}$. (d) Nyquist plots. 


\section{Conclusions}

In summary, we successfully synthesized a new kind of soft carbon and hard carbon composite nanofibers via simple electrospinning and heat treatment. The composite nanofibers web was flexible and was studied as free-standing and binder-free anodes for Li-ion batteries. The CNFs shows good cycling and high rate performance and make it a promising candidate anode material for lithium-ion batteries application in high power electronics.

\section{Acknowledgements}

This research was supported by the National High Technology Research and Development Program of China (863) (2013AA050905, 2014AA052301), the National Nature Science Foundation of China (51172160) and NSF of Tianjin City (14RCHZGX00859, 14JCTPJC00484).

\section{References}

[1] Tarascon JM, Armand M. Nature 2001;414:359-67.

[2] Yu Y, Gu L, Wang C, Dhanabalan A, van Aken PA, Maier J. Angew Chem Int Ed 2009;48:6485-9.

[3] Stevens DA, Dahn JR. J Electrochem Soc 2001;148:A803.

[4] J. R. Dahn. TZ, YingHu Liu, J. S. Xue. Science 1995;270:590-3.

[5] Jo Y-N, Park M-S, Lee E-Y, Kim J-G, Hong K-J, Lee S-I, et al. Electrochim Acta 2014;146:630-7.

[6] Lai C, Zhou Z, Zhang L, Wang X, Zhou Q, Zhao Y, et al. J Power Sources 2014;247:134-41.

[7] Memarzadeh Lotfabad E, Ding J, Cui K, Kohandehghan A, Kalisvaart WP, Hazelton M, et al. ACS nano 2014.

[8] Wang Q, Li H, Chen LQ, Huang XJ. Carbon 2001;39:2211-4.

[9] Zhang B, Xu Z-L, He Y-B, Abouali S, Akbari Garakani M, Kamali Heidari E, et al. Nano Energy 2014;4:88-96.

[10] Yue H, Li F, Yang Z, Tang J, Li X, He D. Mater Lett 2014;120:39-42.

[11] Gibaud A, Xue JS, Dahn JR. Carbon. 1996;34:499-503.

[12] Jin J, Shi Z-q, Wang C-y. Electrochim Acta 2014;141:302-10.

[13] Alcantara R, Madrigal FJF, Lavela P, Tirado JL, Mateos JMJ, de Salazar CG, et al. Carbon 2000;38:1031-41.

[14] Wu Z-S, Ren W, Xu L, Li F, Cheng H-M. ACS nano 2011;5:5463-71.

[15] Li SE, Wang B, Peng H, Hu X. J Power Sources 2014;258:9-18. 